\title{
Tangence
}

\section{Postmodernisme, multiculturalisme et political correctness}

\section{Régine Robin}

Numéro 39, mars 1993

La fiction postmoderne

URI : https://id.erudit.org/iderudit/025749ar

DOI : https://doi.org/10.7202/025749ar

Aller au sommaire du numéro

Éditeur(s)

Tangence

ISSN

0226-9554 (imprimé)

1710-0305 (numérique)

Découvrir la revue

Citer cet article

Robin, R. (1993). Postmodernisme, multiculturalisme et political correctness.

Tangence, (39), 8-20. https://doi.org/10.7202/025749ar d'utilisation que vous pouvez consulter en ligne.

https://apropos.erudit.org/fr/usagers/politique-dutilisation/ 


\title{
Postmodernisme, multiculturalisme et political correctness
}

\author{
Régine Robin
}

Commençons par deux rappels qui n'ont rien d'anecdotiques. Après un séjour universitaire aux États-Unis et au Canada, Luc Ferry prend la plume et écrit dans L'express du 12 octobre 1992 :

Un professeur en mission culturelle à l'étranger doit s'attendre à tout. Pourtant, devant présenter devant le public très largement estudiantin des universités de Québec, Montréal et Ottawa, un exposé aussi peu audacieux que "La Déclaration des droits de l'homme de 1789", je fus éberlué quand des collègues canadiens au regard fuyant et à la mine embarrassée objectèrent que cet intitulé faisait scandale. Pour m'épargner des ennuis avec les puissants groupes féministes, on avait d'ores et déjà fait imprimer les affichettes annonçant la conférence sous le titre "politiquement correct" de "Déclaration de droits humains de 1789 \%.

Pris de l'envie de rire et celle de claquer la porte, mais tenant compte des 6000 kilomètres parcourus, j'acceptai cette formulation étrange, mais commençai mon exposé par une condamnation argumentée du petit attentat auquel se livraient mes hôtes. Car il relève, qu'ils le veuillent ou non, du terrorisme intellectuel. En admettant même que des "inégalités" se soient, au fil des siècles, inscrites dans le langage, en l'espèce dans cette langue française qui désigne par l'"homme "l'être humain en général, seuls les régimes staliniens ou fascistes se sont avisés de réécrire l'Histoire. Et la Déclaration des droits de l'homme, qui en fait partie, n'a pas à être débaptisée par quelques écervelés ${ }^{1}$.

Je tire mon second exemple (mais bien entendu, il y en aurait mille) d'un article de la Gazette de Montréal en date du 26 septembre 1992, à propos d'une querelle concernant la mise en scène d'une pièce australienne dont l'intrigue se passe en Tanzanie. Un certain metteur en scène la monte donc, mais il rencontre

1 Luc Ferry, "La dictature de la "Novlangue" ", L'express, 9 octobre 1992, p. 56. 
toutes sortes d'obstacles jusqu'à ce qu'il découvre qu'il existe dans les milieux anglophones du théâtre à Montréal et plus largement au Canada un consensus non écrit selon lequel seuls des Noirs peuvent monter une pièce noire, ou si le metteur en scène est Blanc, il lui faut l'accord d'un membre représentatif de la communauté noire et ainsi de suite. Une femme ne pourrait parler que de femmes, un homme que d'hommes, et encore de sa propre couleur, un Italien ne pourrait représenter que des Italiens, etc. On n'avait pas encore vu un tel apartheid culturel se mettre en place. Le Conseil des arts a trouvé un terme pour désigner le crime: il s'agit de l'appropriation culturelle. L'auteur de l'article faisait remarquer qu'à ce compte-là, on aurait dû interdire les spectacles d'Ariane Mnouchkine, en tournée à Montréal précisément. En portant à la scène les Atrides d'Eschyle, elle dirige des acteurs de pays non européens en leur laissant leur accent en français, emprunte au théâtre indien et japonais, mélange les musiques, revivifie les Classiques par cette hybridité culturelle et cette hétérogénéité en se moquant bien de faire estampiller ses emprunts.

Que se passe-t-il donc? et en quoi ces phénomènes ineptes relèvent-ils de quelque chose qui aurait à voir avec le postmodernisme?

Au delà des définitions multiples qui ont été données du postmodernisme, je ne voudrais retenir que ce qui est directement utile pour mon propos, car la notion balise tantôt une redéfinition des rapports économiques et sociaux, tantôt une nouvelle donne esthétique. Le postmodernisme serait le terme renvoyant à une sociêté où le marché aurait tout dominé, tout englobé, où la rationalité instrumentale et la médiatisation, la communication auraient tout submergé; cette structure universelle de marché s'articulant sur une vie sociale ghettoïsée, communautarisée ; chaque groupe restant enfermé dans sa différence, ses droits, ses comportemements, sa culture. Plus d'universalisme, plus de monopole de sens, plus de "grands récits"; l'instrumentalité s'est séparée du sens. Comme l'écrit Alain Touraine :

Mais le succès de la critique postmoderniste ne dispense pas de chercher une nouvelle définition de la modernité qui repose sur l'autonomie relative de la société et des acteurs. Car il est impossible d'accepter aisément que leur dissociation soit complète, comme le suggère la coexistence en cette fin de siècle du néolibéralisme et du postmodernisme, dont l'un décrit une société 
10

réduite à n'être qu'un marché sans acteurs (c'est-à-dire où les comportements sont prévisibles à partir des lois du choix rationnel) et dont l'autre imagine des acteurs sans système, enfermés dans leur imaginations et leurs souvenirs ${ }^{2}$.

Sur le plan esthétique, le postmodernisme a rompu avec l'avant-garde et son dispositif de vision révolutionnaire du monde ou à tout le moins subversif ou transgressif. À partir de là on peut opposer deux types de postmodernisme ou deux tentations dans la littérature et l'art contemporains (peinture, photographie, installations, mais aussi bien architecture et musique). La première, je l'appellerai le postmodernisme de la simulation ou du simulacre. Il s'agit de pousser à l'extrême cette dissociation entre les acteurs et le système dont parlait Alain Touraine, de pasticher jusqu'au kitsch, jusqu'à une poétique de la déglingue. On verra à ce sujet aussi bien la surenchère du kitsch chez Jeff Koons, le modèle de Las Vegas proposé par Robert Venturi et son école, et toute l'esthétique qui se réclame de la pub et de Disneyland. Participent de ce modèle, les simulacres ou littérature artificielle (le nouveau Scarlett par exemple) ou cette mémoire de synthèse produite par les "rewriting" et les "remake", la récriture de l'Histoire à l'Est accompagnant la chute comme un château de cartes de l'Union soviétique, du mur de Berlin et de tous les régimes dits satellites. Participe également de ce modèle l'identité vide (comme un écran vide), du sujet trop éclaté, trop sollicité. C'est que la tendance à l'éclatement, à la pulvérisation, à reproduire de l'identique et donc de l'anonymat s'accentue en tous points, au delà même de l'existence ou de la constitution de grandes entités économico-politiques. La prévalence des images, du monde des images (y compris les vidéo-games et les images de synthèse), l'importance du décor du kitsch, du clinquant, l'importance de l'image, du donné-à-voir, a vidé l'intériorité, l'intime, le rapport de soi à soi.

Deux figures de cette dissolution me paraissent emblématiques. D'une part Zelig, le personnage du film de Woody Allen. Il n'est rien, un caméléon potentiel. Il se constitue en fonction de ce qu'il décode du désir de l'autre ou de l'être de l'autre. Il grossit en face d'un gros, grandit en face d'un grand, etc. Trace vide qui ne peut exister qu'en fonction du donné à voir de l'autre. N'être rien ou potentiellement être tout. En face, 
Michael Jackson que Jean Baudrillard appelle l'androgyne parfait ${ }^{3}$. Tout a été refait sur le corps de Michael Jackson. Ses cheveux ont été lissés, sa peau a été quelque peu blanchie, sa voix a été transformée de telle façon que, tendant au "neutre", chaque adolescent puisse s'identifier à sa personne. N'être rien, au plus près des traits de tous. N'être qu'une image potentielle, une image virtuelle, n'est-ce pas une des définitions du postmodernisme que cette labilité, ce potentiel, ce déplacement perpétuel, cette disponibilité qui ne peut s'actualiser que dans le présent, amnésique, immatériel, comme un écran d'ordinateur non relié à une imprimante. Participent encore à cette logique, les Non-Lieux étudiés recemment par Marc Augé, ces lieux identiques les uns aux autres, totalement déracinés comme les aéroports, les grandes chaînes d'hôtels, les supermarchés, les stations d'essence, les parkings, les autoroutes, etc. Cette esthétique du kitsch et du banal s'accompagne très bien d'un retour du lisible au sens où l'entendait Roland Barthes, et donc d'un retour des formes traditionnelles d'écriture. En étudiant une ou deux années de production romanesque en France (1987, 1991), on pourrait montrer ce phénomène. Il en serait de même pour l'architecture qui se complaît également (quand elle ne prône pas purement et simplement le kitsch) dans des formes de retour et de réinscription du monumental des années trente (Ricardo Bofill).

C'est pourquoi ne sont contradictoires qu'en apparence le kitsch version Disneyland et les nationalismes de carton-pâte dont nous sommes les témoins aujourd'hui, nationalismes de "remake", quelque tragiques qu'ils soient.

En face de ce postmodernisme du vide, ou de la résignation aux structures du marché ou des retours les plus divers, je voudrais mettre l'accent sur une autre acception du postmoderne, beaucoup plus intéressante, celle qu'on pourrait appeler le postmodernisme $d u$ trouble. Dans la littérature et dans l'art, dans la vie sociale, cette attitude ne vise ni à subvertir, ni à se résigner mais à contourner, à mettre à distance, à parodier, à déplacer, à décentrer de façon à susciter une inquiétante étrangeté, une attitude critique, de façon à faire des identités troublées, hybrides qui sont les nôtres aujourd'hui, une valeur positive, créatrice, et

3 Jean Baudrillard, La transparence du mal, Paris, Galilée, 1990. 
non inhibitrice. Comme le fait remarquer Guy Scarpetta: "Ni nostalgie du combat, donc, ni complaisance cynique ou désabusêe: le trouble, c'est ce qui nous reste quand le combat n'a plus de sens (ou ce qui revient au même, n'est plus désirable) et quand l'acceptation veule commence à nous écourer "4 .

Je laisse à d'autres l'examen de la tendance du postmodernisme qui ne fait pas l'économie de la complexité, de l'hétérogénéité, qui participe du paradigme du trouble, je n'envisage ici que celle qui simplifie, caricature et signale une véritable "défaite de la pensée " pour reprendre le titre d'un livre d'Alain Finkielkraut. Comme toujours au point de départ, les meilleures intentions. Il fallait bien entamer le vieil ethnocentrisme qui faisait de la civilisation occidentale "la" civilisation, et mesurer les limites d'un universalisme qui avait cautionné le colonialisme et, sur un autre plan, le stalinisme. Pourtant, la force de la pensée universaliste, quelles que soient ses variantes, avait été assez grande pour asseoir définitivement en France le modèle républicain, mobiliser les masses du monde entier pendant plus d'un siècle au nom du socialisme, permettre aux États-Unis de mener la lutte pour les droits civiques et l'intégration raciale. C'est au nom de l'universalisme et des droits de l'homme que les luttes de décolonisation commencent, en retournant l'idéologie du colonisateur contre luimême, etc., mais quelque chose qui a commencé comme une réflexion critique et salutaire, comme une remise en question nécessaire, a dérapé, et le "politiquement correct" rend compte de ce dérapage.

Quatre grandes époques et entités balisent l'attaque contre l'universalisme abstrait des droits de l'homme, du rationalisme, de la croyance aux progrès et à la promotion de l'individualisme contre tous les holismes.

De Herder à Burke, du second Fichte à Joseph de Maistre, l'attaque contre la Révolution française est générale. Il faut opposer à la vulgate des Lumières l'esprit des peuples, leur unicité, leur irréductibilité, leur caractère propre. À la théorie artificielle du contrat, il faut opposer la tradition, le lent mûrissement des lois ancestrales, les corps et les privilèges, à la liberté, les libertés et à l'esprit républicain, les religions et les monarchies. Herder va

4 Guy Scarpetta, «le trouble*, dans Art Press, hors-série n 13, 1992 : numéro spécial 20 ans, l'Histoire continue, p. 134. 
même jusqu'à réhabiliter les préjugés qui avaient été la bête noire des Révolutionnaires de 1789. On sait qu'au lendemain de la défaite de Napoléon et avec la promotion du Romantisme allemand, cet ensemble d'axiomes a fait merveille comme les fameux chassepots. Toute l'Europe centrale, orientale, les Balkans se draperont dans les plis de ce drapeau qu'on peut appeler culturaliste avant la lettre.

Dans le reflux de l'ethnologie évolutionniste et impérialiste, une autre anthropologie prend naissance. Elle associera aussi bien les tenants du fonctionnalisme que ceux du culturalisme proprement dit, que l'incontournable Claude Lévi-Strauss. Tous pourraient souscrire, au delà d'énormes différences théoriques et méthodologiques, à ces propos de Lévi-Strauss:

Mais si l'humanité ne se résigne pas à devenir la consommatrice stérile des seules valeurs qu'elle a su créer dans le passé, capable seulement de donner le jour à des ouvrages bâtards, à des inventions grossières et puériles, elle devra réapprendre que toute création véritable implique une certaine surdité à l'appel d'autres valeurs, pouvant aller jusqu'au refus sinon même à leur négation. Car on ne peut à la fois se fondre dans la jouissance de l'autre, s'identifier à lui, et se maintenir différent. Pleinement réussie, la communication intégrale avec l'autre condamne, à plus ou moins longue échéance, l'originalité de sa et de ma création. Les grandes époques créatrices furent celles où la communication était devenue suffisante pour que des partenaires éloignés se stimulent, sans être cependant assez fréquente et rapide pour que les obstacles, indispensables entre les individus comme entre les groupes, s'amenuisent au point que des échanges trop faciles égalisent et confondent leur diversité 5.

Victoire du relativisme culturel qui, s'il marque une avancée réelle par rapport à tous les ethnocentrismes et toutes les dominations, se retourne très vite en un enfermement.

Dans le cadre même de la sociologie, avec P. Bourdieu et avec bien d'autres, le relativisme culturel a pris d'assaut ce qui pouvait rester de "culture légitime" pour la démystifier. Plus de "high" et de "low" culture, mais une distribution sociale des lectures des habitudes culturelles, des automatismes, une distribution sociale des goûts qui confère la même valeur à Madame Bovary et à n'importe quel roman Harlequin, la hiérarchie n'étant

5 Claude Lévi-Strauss, Le regard éloigné, Paris, Plon, 1983, p. 47-48. 
qu'une intériorisation des normes arbitraires. Toutes les formes culturelles se valent, toutes les cultures se valent; seules président à leur hiérarchisation les instances de légitimation, c'est-à-dire une forme de pouvoir.

Le combat même de la décolonisation dans les années cinquante, avec Fanon et Senghor, en prônant "la négritude ", l'exaltation contre le colonisateur de l'extrême singularité, rejette toute promotion de l'individu au profit de la multitude, de la communauté.

Une certaine interprétation, en particulier en Amérique du Nord, de Mikhail Bakhtine et de son dialogisme, de Michel Foucault montrant que le pouvoir n'est pas uniquement central mais peut se trouver disséminé dans des formes, des lieux, des institutions, de Jacques Derrida surtout et tout le mouvement déconstructionniste disant qu'il n'y a pas de vérité ultime du texte mais des points de vue qui en creusent de nouveaux, des interprétations auxquelles il manque toujours une clé, des éléments qu'on peut mettre en avant en déconstruisant le texte qui de ce fait ne ressortit pas à un régime de vérité, mais à une infinité de déploiements potentiels, etc.

On voit bien ce qui est en cause lorsque ces idées qui relèvent d'époques et de cadres épistémologiques distincts se mettent (en se simplifiant) à circuler dans le discours social en se combinant étrangement: relativité des cultures et des productions culturelles, de tous les biens symboliques, absence de régime de vérité, nécessité du dialogisme, du carnavalesque, de la parole populaire, promotion du communautaire au détriment d'un universalisme et d'un individualisme qui débouche aujourd'hui dans nos grandes villes sur l'anonymat, l'atomisation, la solitude et le contraste entre les valeurs proclamées: Liberté, Égalité, Fraternité, pour ne prendre que l'exemple français, et la réalité quotidienne du chômage, du racisme, de l'exclusion. Le repliement sur le moi engendre le narcissisme généralisé de ce que Charles Taylor appelle la culture de "l'authenticité "6, et Gilles Lipovetsky, l'ère du vide 7 .

Si l'on met à part ce qui a suivi l'effondrement du communisme et la tragédie des Balkans installés dans une guerre sans

6 Charles Taylor, Grandeur et misère de la modernité, Montréal, Bellarmin, 1992.

7 Gilles Lipovetsky, L'ère du vide. Essais sur l'individualisme contemporain, Paris, Gallimard, 1983. 
nom, c'est aux États-Unis à l'heure actuelle que la contestation du modèle universaliste a été la plus forte et a débouché sur ce phénomène curieux que constitue le politically correct, à la croisée de plusieurs mouvements: le relatif échec de l'intégration des Noirs et de l'affirmative action, les conséquences du repli sur soi de la culture narcissique, l'extrême judiciarisation de la société américaine et le mouvement d'affirmation de soi de toutes les minorités et de toutes les communautés après des années de conservatisme et de reaganisme qui donnaient le sentiment que tout projet social collectif d'ensemble comme le Rêve américain n'avait plus aucune consistance.

Cela a donné naissance à un etbnic revival généralisé, à une mosaïque de définitions identitaires collectives qui sont toutes en concurrence entre elles, toutes en lutte pour la reconnaissance culturelle, avec au moins, jusqu'à l'élection de Bill Clinton qui a tenté de revitaliser "le Rêve américain", toutes, dans l'abandon d'un projet culturel commun transverse. On ne s'appesantira pas ici sur l'histoire même du mouvement du politically correct, depuis les remises en question des curricula dans les universités jusqu'à cette censure armée des meilleures intentions qui oblige à modifier le vocabulaire en usage et aboutit à une véritable langue de bois. On trouvera beaucoup d'ouvrages sur ce point précis, mon propos n'est pas là. Disons que ce qui est en jeu, ce sont deux conceptions du multiculturalisme, et donc, là encore, du postmodernisme. Melting pot ou salad bowl?

Comme une salade, les États-Unis sont faits de différents ingrédients de peuples et de cultures, explique un nouveau manuel d'Histoire. Bien qu'ils soient mélangés, ces ingrédients restent distincts. Et, comme chaque composante dans un saladier comporte une saveur particulière, chaque contribution culturelle ajoute à la variété et à la diversité de la vie américaine. ${ }^{8}$

Si la remise en question d'un ethnocentrisme trop centré sur les Classiques et l'héritage européen était un mouvement positif, si le féminisme avait au départ mis l'accent sur les vrais problèmes qui affectent en permanence la société américaine, la dérive de ces mouvements ne s'est pas fait attendre. D'abord, s'est imposé ce que j'appellerai le modèle Blade Runner, d'après le célèbre film de Ridley Scott. Los Angeles au XXI ${ }^{e}$ siècle, plus ou

8 Cité d'après Annick Cojean, *Désarrois américains", Le monde, 30 octobre 1992, p. 6. 
moins colonisé par les Japonais. Chacun vit comme il peut et le seul transverse est un sabir où l'on reconnaît encore quelques mots d'anglais. Chacun "sa culture", son propre héritage culturel, chacun ses codes sociaux, chacun son propre système symbolique. On peut ainsi relire l'histoire du monde à l'image de l'africanité; c'est le cas de l'école de pensée fondée par Molefi Asante par exemple. Dans cette perspective, à l'école, les professeurs viennent avec des costumes africains, les chants enseignés sont de type traditionnel, l'histoire enseignée montre que tout vient d'Afrique via l'Égypte. African-American is beautiful, et en face de ce racisme effréné dont la société américaine ne vient pas à bout, on voit se développer un "contre-racisme" parfaitement symétrique du premier et dont les porte-parole très différents l'un de l'autre pourraient bien être Leonard Jeffries et Spike Lee. Tel groupe de Native-Americans fait pression pour que soit reconnu explicitement le rôle joué par le modèle iroquois sur la Constitution des États-Unis, pour ne pas parler de ce à quoi la célébration de l'arrivée de Christophe Colomb en Amérique a donné lieu en 1992. Une Amérique des tribus avec, au-dessus, une classe politique en porte-à-faux. La fin de l'universalisme a été ainsi la promotion des minorités, du droit des minorités à s'affirmer culturellement comme telles. Cela a donné la Identity Politics $^{9}$. Comme le résume très bien Michel Feher:

Il s'agit donc de promouvoir la solidarité communautaire, tant en mobilisant les membres du groupe autour des injustices spécifiques qu'ils subissent, qu'en les incitant à découvrir ou à retrouver leur culture propre, étouffée ou tout le moins marginalisée par la culture dominante. Les politiques identitaires défendues par les différentes composantes de la coalition multiculturaliste comprennent par conséquent deux volets distincts: d'une part, chacune œuvre à la réparation des méfaits causés à la population qu'elle représente - par le racisme, le sexisme, ou l'homophobie; d'autre part, ces mêmes politiques se donnent pour objectifs la réappropriation et le contrôle par chaque communauté de son identité collective; aussi bien pour l'arracher aux stéréotypes infamants produits par l'idéologie dominante, que pour la sauver de l'effacement auquel elle est promise dans le cadre du modèle libéral d'intégration... Au célèbre melting pot,

9 Sur ce sujet voir entre autres Dinesh D'Souza, Mlliberal Education: the Politics of Race and Sex on Campus, 1991; Beyond PC: Toward a Politics of Understanding, Patricia Aufderheide (éd.), 1992; Debating PC, Paul Berman (éd.), 1992. 
dont la minorité noire a toujours été exclue et qui ne corrige en rien ni l'assujettissement des femmes aux hommes ni l'ostracisme persistant qui frappe les homosexuels, les promoteurs de la société multiculturelle substituent le modèle de la mosaz̈que qui s'efforce de combiner la conquête de l'égalité entre les individus avec le respect des différences de culture entre les multiples communautés qui composent la société américaine ${ }^{10}$.

Le problème dans ce type d'acception, c'est précisément cette tension impossible à maintenir entre les droits individuels et la lutte pour l'égalité des chances et la promotion de droits collectifs sans projet social transverse. Il y a des sociétés où ces problèmes, du moins jusqu'à présent, se sont posés tout autrement, comme dans le modèle français, mais il est vrai que nous étions loin du postmodernisme.

Ceci pose le problème du politique, de la cité, de la citoyenneté et du mode d'intégration-assimilation des étrangers à tel ou tel ensemble national. Je dis bien intégration-assimilation pensant que le tabou qui a frappé le terme "assimilation" relève d'une mauvaise querelle. Je suis sur ce point en accord total avec Dominique Schnapper qui développe longuement sa question dans son dernier livre ${ }^{11}$ ainsi qu'avec Stéphane Beaud et Gérard Noiriel qui en ont fait une récente mise au point ${ }^{12}$.

L'intégration-assimilation désigne les processus par lesquels les individus acquièrent peu à peu les codes, les normes de la société dans laquelle ils vivent ou sont amenés à vivre, et ce, quelle que soit la politique officielle suivie, ce processus échappant aux volontés politiques et hégémonies idéologiques. La culture est un processus, un mode d'acculturation, processus, comme le dit Schnapper, "au cours duquel les individus immigrés ou non - acquièrent, perdent, renouvellent, élaborent, interprètent et réinterprètent des éléments divers. Toute constitution d'identité est un processus dynamique d'élaboration culturelle. C'est pour marquer ce caractère dynamique que le terme d'acculturation me paraît préférable à celui de culture" 13 .

10 Michel Feher, "Le retour du mot en "L" *, Libération, 2 novembre 1992, p. 6.

11 Dominique Schnapper, La France de l'intégration, Paris, Gallimard, 1991.

12 Stéphane Beaud et Gérard Noiriel, "Penser l'intégration des immigrés *, dans Face au racisme, sous la direction de P.A. Taguieff, Paris, 1991, t.2.

13 Schnapper, op. cit., p. 87. 
18

Dans le processus d'assimilation-intégration, il faut distinguer deux processus distincts. Le premier a trait à la dimension psychosociologique de l'assimilation et renvoie au fait que quels que soient les déchirements culturels, la seconde génération s'assimile aux normes dominantes partout. Beaud et Noiriel écrivent:

La facilité avec laquelle la plupart d'entre eux assimilent la langue française même quand leurs parents ne la parlent pas a ici valeur emblématique et prouve l'inanité de toute l'argumentation en terme de "distance culturelle" opposant une prétendue "culture européenne" aux cultures du tiers-monde. Dans cette perspective, tous les enfants qui sont socialisés dans le pays d'accueil, par définition "assimilent" la culture nationale (et sont donc "assimilés" par elle), selon des modalités qui reproduisent la diversité propre à la société considérée (selon le milieu social, la région où ils vivent, etc.), l'attachement au "pays d'accueil", le sentiment d'appartenance qu'ils éprouvent à son égard, même quand ils le critiquent... ${ }^{14}$.

Le second aspect du processus d'assimilation-intégration a trait à la participation à la vie collective: modalités d'accès au marché du travail, intégration aux formes de sociabilité, participation à la vie politique. Si bien que Beaud et Noiriel concluent leur analyse par la réflexion suivante:

$\mathrm{Au}$ risque de décevoir, nous soulignerons en premier lieu que le "problème " de l'assimilation ne peut se résoudre uniquement à coup de mesures politiques et même que certaines mesures politiques irréfléchies peuvent aller à l'encontre de l'objectif proclamé. L'histoire de France démontre que les précédentes "deuxièmes générations" (que l'on disait déjà difficilement "assimilables") se sont intégrées sans qu'aucune politique spécifique ait été mise en œuvre par les pouvoirs publics. De même on constate [...] que malgré les différences de système politique en France et aux USA, le processus d'assimilation des immigrants depuis un siècle [...] s'est accompli de façon aussi efficace dans les deux cas, en fonction des traditions propres à chacune de ces deux sociétés nationales ${ }^{15}$.

L'égalité du citoyen n'a jamais entraîné l'homogénéité des conditions sociales connotée par le concept d'assimilation. L'assimilation ne renvoie pas à une homogénéité mais au cantonnement

14 Beaud et Noiriel, op. cit., p. 277.

15 Ibid., p. 279. 
dans la sphère du privé (à partir de la loi sur les associations de 1901 en particulier) de toutes les pratiques communautaires liées à la religion, à l'origine ethnique ou nationale. L'égalité des citoyens implique une richesse de la vie associative et non pas l'inverse.

Je crois donc pouvoir poser qu'il faut préférer le peuple des citoyens au peuple des ancêtres supposés, le demos à l'ethnos, la société civique aux débordements de la société civile ou à sa prééminence, ce qui rassemble plutôt que ce qui singularise, et l'horizon d'un projet de société plutôt que la panique identitaire devant la peur de la perte de soi. Aujourd'hui, toutes les sociétés sont menacées par l'ethnicisme, c'est-à-dire par l'absolutisation des différences.

Mais la France n'est pas l'Amérique du Nord et aujourd'hui elle est aussi confrontée au phénomène de la déchirure du tissu républicain, au postmodernisme du simulacre et des tribus, au multiculturalisme.

Il y a cependant une seconde acception possible du postmodernisme et donc une autre façon de penser le multiculturalisme. C'est la problématique du trouble comme nous l'avons déjà mentionné. Dans cette seconde acception, le phénomène du politically correct n'est pas possible car on sait bien que ce qui fait problème c'est le réel, non sa transcription langagière, et qu'il n'est pas bon de faire semblant que les préjugés ont été vaincus alors qu'ils sont simplement censurés, et que la confrontation au mal vaut mieux qu'une société de boyscouts où, de temps en temps, le passage à l'acte prend des formes monstrueuses faute d'avoir pu se mettre en mots. Tout simplement, la réappropriation culturelle est une illusion dangereuse sur plusieurs plans : elle nie l'Histoire, les défaites et les nouveaux arrangements identitaires, les hybridités culturelles, les emprunts, les détournements et les retournements, le réel tout simplement; elle naturalise les faits de culture en obligeant les individus à entrer dans une case définitoire, à dire ce qu'ils sont alors que le projet humain ne peut jamais se laisser enfermer dans une classification, fût-elle identitaire; elle essentialise l'être, elle substantifie l'identité. Elle mène, armée des meilleures intentions, au racisme à l'envers, à l'apartheid consenti, à l'enfermement identitaire. Dangereuse encore, parce que comme un certain nombre d'historiens l'ont démontré, l'ancestralité est un leurre, elle est récente, et que s'inventer son passé, le modeler en fonction des besoins idéologiques du moment mène à ce qui se passe en Bosnie où chaque groupe se 
bat au nom de sa "culture", de son "passé", les passés étant incompatibles les uns avec les autres, bien entendu. C'est s'inventer un mythe de l'origine, un paradis perdu toujours à reconquérir et à reconquérir autrement que dans le fantasme, la littérature, l'art, à reconquérir dans le réel...

Dangereuse encore parce que fondée sur la différence absolutisée et non sur l'altérité. Comme le dit Baudrillard:

Tout se parle aujourd'hui en termes de différences. Mais l'altérité n'est pas la différence. On peut même penser que c'est la différence qui tue l'altérité. Quand on ventile le langage dans un système de différences, quand on réduit le sens à n'être qu'un effet différentiel, on tue l'altérité radicale du langage, on met fin au duel qui est au cœur du langage, entre lui et le sens, entre lui et celui qui parle, on élimine ce qui en lui est irréductible à la médiation, à l'articulation, au sens, et qui fait justement que, dans sa radicalité, le langage est autre que le sujet... ${ }^{16}$.

Une autre acception du postmoderne et du multiculturalisme chercherait à articuler la nécessaire posture universaliste de l'égalité juridique et un maintien des potentialités des multiplicités présentes sans les figer. Est-ce ce que le nouveau Président des États-Unis avait en tête en parlant de "réinventer l'Amérique"? Non pas un retour à une homogénéité culturelle qui, en Amérique, n'a jamais existé, non pas un retour à un détestable ethnocentrisme, mais à une forme de souplesse et au maintien de la complexité dans le tressage de la multiplicité culturelle. Si cela était, pourrait alors s'ouvrir une nouvelle époque, un nouvel espoir où la critique libérale de l'authenticité et du relativisme rencontrerait la critique multiculturaliste de la neutralité et de l'universalisme libéral, dans un débat toujours tendu, assurément interminable, mais incontestablement fécond ${ }^{17}$.

Nous n'aurions plus peur du postmodernisme.

16 Jean Baudrillard, op. cit., p. 131-132.

17 Michel Feher, op. cit., p. 10. 